\title{
Novel Fibrillar Structure in the Stroma of Murine Cornea
}

\author{
Ali R. Behzad ${ }^{1}$, Evelyn Brown ${ }^{2}$, Nancy Shenoi ${ }^{2}$, Margaret Gondo ${ }^{2}$, Alan R. Burns ${ }^{2}$ and Samuel \\ Hanlon $^{2}$ \\ ${ }^{1}$ Advanced Nanofabrication, Imaging and Characterization Lab, King Abdullah University of Science \\ and Technology, Thuwal, Kingdom of Saudi Arabia. \\ ${ }^{2}$ College of Optometry, University of Houston, Houston, United States of America.
}

As the main refracting element of the eye, the cornea is necessarily transparent and appropriately shaped in order to project a focused image onto the retina. Transparency is determined by the ultrastructural organization which must be maintained and restored, if possible, in the event of injury. The unique qualities of the cornea and its importance for vision have fueled extensive research into its physical characteristics and molecular activities. The purpose of the present study was to use serial block-face sectioning and computerized 3D reconstruction to evaluate the organization of a novel filamentous structure within the corneal stroma. In most species the stroma constitutes the bulk of the total corneal thickness and is therefore critical for maintaining useful vision. The extracellular matrix (ECM) consists primarily of type I collagen, organized into tightly-spaced bundles (lamellae) of parallel fibrils laid down in cross-crossed fashion, and a lesser amount of several other types of collagen as well as proteoglycans. Uniform size and spacing of the collagen fibrils is necessary for transparency (1). Resident keratocytes form an interconnected network of flattened cells with long, slender processes interspersed between the collagen lamellae and are directly responsible for the synthesis and secretion of ECM components (2).

Much of the information about the spatial organization of stromal ECM has been obtained by light microscopy (LM), standard transmission electron microscopy (TEM), scanning electron microscopy (SEM) and atomic force microscopy (AFM). Understanding the supramolecualr architecture of ECM is somewhat limited when using a method that provides only two dimensional ultrastructural information (TEM) or only surface information (SEM and/or AFM). Serial block-face sectioning SEM is a novel alternative approach that allows the determination of spatial distribution and organization of ECM ultrastructural components.

Anesthetized mice (C57BL/6; 8-12 weeks old) received a central $2 \mathrm{~mm}$ corneal epithelial abrasion injury and the animals were euthanized $18 \mathrm{~h}$ later. Excised corneas were fixed, heavy metalcontrasted and embeddd in Durcupan as previously described (3). Serial block-face images of corneal stroma (100 nm intervals between images) were obtained using a Gatan 3 View system mounted in an FEI Quanta FEG 200 SEM. Amira 5.2 software was used for 3D image reconstruction and segmentation.

Figure 1 shows a volume of peripheral corneal stroma (Panel A). The segmented and reconstructed images (Panels B, C and D) reveal a novel structure composed of electron dense filaments that assemble into larger "cables" (100-200 nm in diameter) that course through the stroma. This novel fibrillar plexus appears to form a continuous network, extending from the periphery to the center of the cornea. In addition, this network appears to interface with lymphatic vessels and stromal keratocytes. In summary, we have used serial block-face sectioning SEM and 3D reconstruction to reveal the presence of a novel and extensive network of cables that extend throughout the corneal stroma. While the function and composition of these cables remains to be determined, we propose that they function as mechanical force transducers, capable of responding to physical stress within the cornea. This would be particularly relevant to corneal abrasion injury, when the cornea becomes edematous and swells. We anticipate tension would be exerted on the cables which would be 
conducted to the cable insertion points on the peripheral lymphatic vessels causing them to dilate and thereby aid in the resolution of tissue edema.

References

[1] J.R. Hassell and D.E. Birk, Exp Eye Res. 91(2010), 326-335.

[2] A.J. Quantock and R.D. Young, Dev Dyn. 10 (2008), 2607-21.

[3] A.R. Behzad et al., Proceeding of the $15^{\text {th }}$ European Micrsocopy Congress, 3 (2012), 379-380.

[4] The authors gratefully acknowledge funding from the National Institutes of Health under grant numbers EY17120, and P30EY007551.
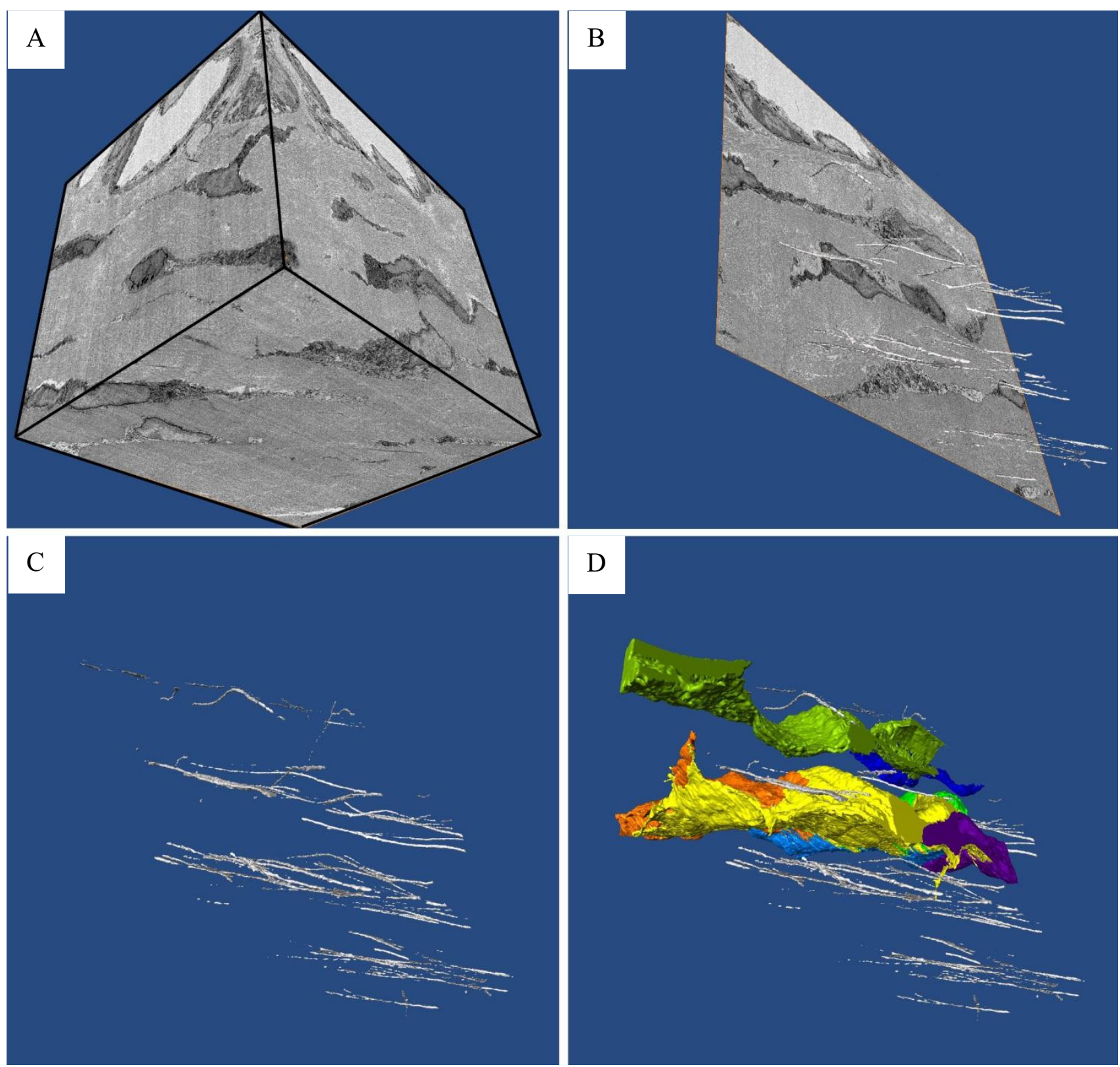

Figure 1. Serial Block-face image reconstruction reveals a network of cables extending throughout the corneal stroma. The volume of cornea scanned $(35 \times 35 \times 35 \mu \mathrm{m})$ is shown as a solid block (A). Stromal elements segmented and reconstructed emerge from the stack of electronmicrographs (B). The reconstructed cables form an intricate network in layers parallel with the corneal surface (C) and are associated with stromal cells (D). Cells seen in (D) are keratocytes (yellow, orange, light blue, dark green, and purple), a neutrophil (light green), and a macrophage (dark blue). 